\title{
Wastewater mediated activation of micromotors for efficient water cleaning
}

(Supporting Information)

Sarvesh Kumar Srivastava*, Maria Guix, Oliver G. Schmidt

Institute for Integrative Nanosciences, IFW Dresden, Helmholtzstraße 20, 01069 Dresden, Germany

* sarvesh.kumar@,ifw-dresden.de 


\section{Material and methods}

Fabrication of rolled-up thin film:

Microjets for the 4-NP degradation experiments were fabricated by rolling up metal thin films evaporated by electron beam deposition technology on defined photoresist micropatterns. Glass wafers $18 \times 18 \mathrm{~mm}$ in size were cleaned with acetone and 2-propanol by sonication for $2 \mathrm{~min}$ in each solvent. The glass wafers were dried and baked at $120^{\circ} \mathrm{C}$ for 2 min. Coating with positive photoresist ARP-3510 was carried out on a spin-coater at $3500 \mathrm{rpm}$ for $35 \mathrm{~s}$. The samples were postbaked at $90{ }^{\circ} \mathrm{C}$ for $3 \mathrm{~min}$. Exposure to ultraviolet light through a mask of $50 \times 50 \mu \mathrm{m}$ squared structures for $7 \mathrm{~s}$ with a Karl Suss MA56 mask aligner leads to photolithographic patterning of the photoresist. The samples are developed in AR 300-35/water (1:1) solution for $50 \mathrm{~s}$ and dried subsequently. Metal evaporation of $5 \mathrm{~nm}$ each of Ti:Fe: $\mathrm{Cr}$ at $75^{\circ}$ and a rate of $3 \AA / \mathrm{s}, 1 \AA / \mathrm{s}$ and $0.5 \AA /$ s respectively was conducted on patterned wafers by using an Edwards e-beam.

Next, silicon grid containing roll-up thin films were cut into sixteen equal parts of dimensions of $0.013 \mathrm{~mm}^{2}$ with wafer dicing machine for its subsequent functionalization. Each of these smaller pieces contained 50 number of unrolled thin metal films for subsequent functionalization.

Growth of catalytically active Pd particles: Microjet

Palladium chloride $\left(\mathrm{PdCl}_{2}\right)$, L-Tryptophan (Trp) and Tetrahydrofuran (THF) were obtained from Sigma-Aldrich (Germany) and were used directly without pre-treatment. Aqueous stock of 10\% THF (v/v) was prepared by mixing thoroughly with deionized water. To prepare the $\operatorname{Pd}(\mathrm{II})$ ionic solution $(1.5 \mathrm{mM})$, pre-weighed $\mathrm{PdCl}_{2}$ was introduced into a clean glass voil and $20 \mu \mathrm{L}$ of conc. $\mathrm{HCl}$ was introduced followed by volume make-up by addition of water. Trp aqueous stock 
solution $(0.1 \mathrm{M})$ was prepared by introducing pre-weighed quantity in ultrapure water and stirred until dissolved.

Pd catalyst growth solution comprising $1 \mathrm{ml}$ each of Pd (II) stock solution and THF in $40 \mathrm{ml}$ of ultrapure water. Finally, $5 \mathrm{ml}$ of Trp stock solution was added into above mixture to initiate the process of $\mathrm{Pd}$ reduction and was used readily thereafter in order to ensure seeding process happening along the surface of unrolled microtube.

Next, diced grid piece of $0.013 \mathrm{~mm}^{2}$ was coated with $10 \mu \mathrm{L}$ of $10 \%$ THF and heated at $80{ }^{\circ} \mathrm{C}$ till the solvent is evaporated. This followed by drop casting of $10 \mu 1$ of Pd catalyst particle growth solution followed and heating microgrid at $95{ }^{\circ} \mathrm{C}$ over a hotplate till the solvent evaporates. Care should be taken not to heat the grid for long as it causes problems in rolling up of the tubes. This active grid containing $\mathrm{Pd}$ catalyst was given a quick wash in 5\% methanol solution to remove any surface adhered organic impurities followed by Millipore water. Finally, acetone: $\mathrm{H}_{2} \mathrm{O}$ solution (3:1) was used to roll these catalytic microtubes. If required, a higher concentration of acetone can be used for rolling-up assisted with gentle scratching to ensure successful release of microjets from the silicon grid. The $\mathrm{Ti} / \mathrm{Fe} / \mathrm{Pt}$ nanomembranes subsequently roll up into microtubes of $50 \mu \mathrm{m}$ length and about $\sim 8 \mu \mathrm{m}$ in diameter (see video S2 in the SI).

\section{Catalytic degradation of 4-NP}

The reduction of 4-NP by $\mathrm{NaBH}_{4}$ was studied as a model reaction to probe catalytic efficiency of poly-Trp stabilized Pd-micromotors as a heterogeneous systems. Under experimental conditions, reduction does not proceed at all simply with the addition of $\mathrm{NaBH}_{4}$ or $\mathrm{Ti} / \mathrm{Fe} / \mathrm{Cr}$. However, in the presence of Trp-Pd grown tubes, it proceeds to completion with formation of 4-aminophenol (4AP). To study the reaction in a quartz cuvette, 4-NP pollutant mixture was prepared by 
introducing $300 \mu \mathrm{L}\left(10^{-2} \mathrm{M}\right)$ of 4-NP solution in $27.7 \mathrm{~mL}$ of water and $2 \mathrm{ml}$ of $0.1 \mathrm{M} \mathrm{NaBH}$. The final concentration of 4-NP and $\mathrm{NaBH}_{4}$ was found to be $0.001 \mathrm{mM}$ and $0.03 \mathrm{mM}$ respectively. The reaction mixture immediately changed from colorless to deep yellow color $(400 \mathrm{~nm})$ due to the formation of nitrophenolate ion.

Subsequent degradation was evaluated by spectrophotometric studies (Analytik Jena specord 250 spectrophotometer), carried out in a quartz cuvette by taking $3 \mathrm{ml}$ of pollutant mixture and introducing the silicon grid containing rolled-up Pd micromotors. This was also compared with the unrolled Pd deposited thin film (grid only) and also rolled-up but not released Pd-microjets. Finally, control comprised of silicon grid containing rolled-up thin films undergoing same reaction treatment except with the growth of $\mathrm{Pd}$ particles. The reaction constant $(\mathrm{k})$ was determined by measuring the change in absorbance at $400 \mathrm{~nm}$ and calculating its natural logirithimic value over a period of $10 \mathrm{~min}(5 \mathrm{sec}$ intervals $)$ as a function of time.

We noted that the wet-lab method for growing Pd-catalysts and subsequent rolling-up of tubes compromises their rolling efficiency (see video S1 in SI). While isopropanol mediated rolling results in unidirectional rolling and smaller tube-diameter, it blocks the internal cavity of the rolled-up tube with Pd catalyst grown inside. Contrary to this, aqueous acetone mediated roll-up results in larger tube diameter, which can hold the Pd-catalyst inside efficiently without blocking the internal cavity of the micromotors (see video S2 in SI). This is of critical importance as the internal cavity of the rolled-up tube must be open throughout for the nucleation and subsequent bubble generation for assisted propulsion as micromotors. 
Material characterization and media analysis

Scanning electron microscopic pictures were taken with a Zeiss SEM/FIB scanning electron microscope. Also, surface analysis was carried out before and after the reaction from a PHI-5600 photoelectron using (Al)K $\alpha$ radiation with pass energy as $29 \mathrm{eV}$.

A Zeiss axioScope A1 optical microscope with Phantom Miro eX2 high-speed camera from VisionResearch was used to record videos of homogeneous activation and movement of our micromotors at the rate of 50 frames per second (unless otherwise stated). The free software FiJi was used to track particle speed and edit videos and pictures.

We fabricated Pd grown-tubular micromotors which moved in aqueous medium via bubble propulsion. Growth of 100-400 nm of dendrite-shaped Pd was achieved inside the tube enabling catalytic degradation of 4-NP in presence of $\mathrm{NaBH}_{4}$. Upon addition of $\mathrm{NaBH}_{4}$ with 4-NP solution, the color of the reaction solution turned to yellow due to the formation of nitrophenolate ion red-shifting the absorption peak to $400 \mathrm{~nm}$. The color of the solution remained unchanged for several days in absence of a catalyst as observed in other studies. Only upon addition of Pd-grown tubes, the catalytic degradation of 4-NP was initiated resulting in rapid color change from yellow to colorless. Our study provides the first of its kind proof of concept where a catalytic micromotor gets activated in the presence of pollutant mixture and proceeds for its complete degradation. 


\section{XPS}

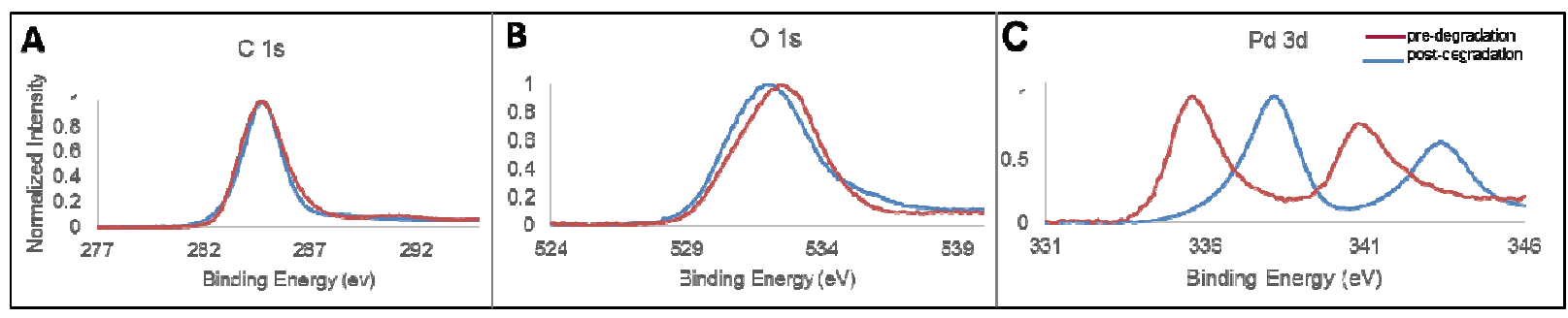

Figure S1. XPS spectra of Pd grown micromotors before (red) and after (blue) degradation:

$$
\text { A) C 1s B) O 1s C) Pd 3d. }
$$

An XPS study was undertaken to analyze surface changes before and after the degradation reaction. Figure 4A shows $\mathrm{C} 1 \mathrm{~s}$ spectra with almost identical overlapping peaks for the C-C interactions at $284.8 \mathrm{eV}$ suggesting a highly stable carbonaceous framework. In this context, our organic layer-supported palladium shows identical properties as actual carbon-layers showing chemical inertness. Similarly, the O1s (figure 4B) spectrum showed a characteristic peak at $\sim 531$ $\mathrm{eV}$ due to the organic $\mathrm{C}-\mathrm{O}$ and $\mathrm{C}=\mathrm{O}$ framework. The associated peak width was broadened and shifted to around $532 \mathrm{eV}$ suggesting formation of oxides which was later confirmed for the Pd catalyst. In figure $4 \mathrm{C}$, the $\mathrm{Pd} 3 \mathrm{~d}$ spectra before and after the reaction were compared. We observed a characteristic $\mathrm{Pd} 3 \mathrm{~d}_{5 / 2}$ peak at $335.8 \mathrm{eV}$ and a $\mathrm{Pd} 3 \mathrm{~d}_{3 / 2}$ peak at $341 \mathrm{eV}$ as reported previously for supported $\mathrm{Pd}$ catalysts. These $\mathrm{Pd} 3 \mathrm{~d}_{5 / 2}$ and $\mathrm{Pd} 3 \mathrm{~d}_{3 / 2}$ peaks shifted to $338.2 \mathrm{eV}$ and $343.5 \mathrm{eV}$ respectively, suggesting surface oxidation of the Pd catalyst after the reaction. We observed that our Pd-micromotors can be effectively re-used thrice for an estimated operating 
time of c.a 30 minutes (three degradation cycles) under the reported 4-NP degradation parameters.

Supporting Videos:

Video S1. Comparative evaluation of the rolling efficiency of the $\mathrm{Ti} / \mathrm{Fe} / \mathrm{Cr}$ thin films by isopropanol and aq. acetone.

Video S2. Rolling-up and releasing of Pd grown catalytic micromotors via aq. acetone mixture.

Video S3. Rolled-up e-beam deposited Pd (10 nm) microtubes in presence of 4-NP reaction mixture.

Video S4. Bubble nucleation and release in Pd-catalytic microtubes in presence of 4-NP reaction mixture (1000 fps)

Video S5. Homogeneous activation of Pd grown micromotors in presence of 4-NP pollutant mixture.

Video S6. Real time visualization (color change from yellow to colorless) of 4-NP degradation by grid only, rolled-up not released and rolled-up released (micromotor). (16x)

Video S7. Single micromotor motion (normal and diluted 4X pollutant mixture) 